\title{
Workshop in Kinderphilosophie: Ich-Du-Wir. Eine philosophische Entdeckung des Anderen
}

\author{
Małgorzata Bogaczyk-Vormayr (Adam Mickiewicz University)
}

\section{Vorbemerkungen}

Der Workshop ist für Kinder im Alter von 9 bis 11 Jahren konzipiert. Allerdings lässt sich dieser durch eine Modifikation der Arbeitsaufträge und eine andere Spieleauswahl leicht auf die Zielgruppe der 7- bis 10-Jährigen bzw. der 11- bis 14-Jährigen ausdehnen. Auch für SchülerInnen der AHS ist die Thematik dieses Workshops geeignet: Hierbei würde der Schwerpunkt mehr auf Textarbeit liegen, auf einer Bearbeitung der o.a. philosophischen Begriffe und auf diversen Diskussionstechniken. Zum Beispiel wäre es möglich, die historischen Kontexte der Entwicklung der dialogischen Philosophie zu beleuchten: Lévinas’ Projekt „Philosophie nach Auschwitz“ - „Philosophie als Ethik“ - „Ethik als Erste Philosophie“).

Geplante Teilnehmerzahl: 12 - 20 Kinder.

1.1. Zweck, Vorhaben, geplante Effekte (didaktische, pädagogische, soziale)

Der Zweck des vorliegenden Projektes ist die Anregung der philosophischen Aktivität bei Kindern, d.h. die Entwicklung der Fähigkeit, Fragen sachlich zu stellen und darüber zu reflektieren, besonders die Fähigkeit, philosophische Fragen zu stellen. Im Mittelpunkt stehen dabei die gemeinsame Bearbeitung der aufgeworfenen Probleme, die Formulierung eigener Ideen zum Thema sowie eine Ermunterung zum philosophischen Gespräch, zum Dialog.

Das Thema dieses Workshops ist die philosophische Kategorie des Anderen. Philosophie soll dabei als Disziplin auftreten, die zwar metaphysische Wurzeln hat, allerdings auch die heutigen sozialen Themen betrifft. Mein Ziel ist es, die Kinder durch geeignete Spiele und Übungen und durch eine Orientierung an deren Sprachniveau, vor allem aber durch die Hervorhebung ihrer ersten, intuitiven Reflexionen deren soziale und kulturelle Akzeptanz der Alterität zu entwickeln.

\subsection{Skizze der Problematik}

Die Dialogphilosophie (Begegnungsphilosophie) gehört zu den bedeutendsten Richtungen in der Philosophie des 20. Jahrhunderts. Sie umfasst Themen aus den Bereichen Ethik, Sozialphilosophie und philosophische Anthropologie, aber auch aus der Metaphysik. Zugleich ist sie eine gänzlich unsystematische 
Richtung, weswegen ich glaube, dass man diese Problematik den Kindern sowohl auf wissenschaftlichem Niveau als auch interessant und eben verständlich näherbringen kann. Das vorgeschlagene Thema finde ich in der philosophischen Lehre für Kinder und Jugendliche besonders wichtig. Ich beabsichtige, die Philosophie des Dialogs zugleich historisch wie auch als eine moderne Quelle für die kulturell-soziale Förderung der Toleranz, der Freiheit, der Menschenrechte zu präsentieren, als Kritik der Xenophobie und des Rassismus.

Wie allgemein bekannt, wurde die Begegnungsphilosophie unter anderem durch Martin Buber, einen deutsch-jüdischen Philosophen, begründet. Ein zentraler Gedanke ist dabei sein Konstrukt der "Grundworte“ Ich - Du. Seiner Meinung nach liegt darin eine von zwei Grundeinstellungen eines Menschen gegenüber der Welt; der Mensch ist konfrontiert mit der Wahl zwischen den Bereichen Ich-Du oder Ich-Es. Gemeinsam mit den Kindern möchte ich erarbeiten, was es bedeutet, einen Menschen als Subjekt vs. als Objekt zu behandeln, was es eigentlich heißt, einer anderen Person zu begegnen, wie die Begegnungen und Relationen mit den Menschen unseren Charakter und unsere Werte formen. „Wie betrachten und empfinden wir die Welt?", werde ich die Kinder fragen. Wir sehen und gebrauchen verschiedene Dinge in der Welt, aber nicht alles ist ein "Ding“. Wir gehen auf verschiedene Arten mit verschiedenen "Wesen“ um - wie? Weiters werden die Kinder mit der „Epiphanie des Antlitzes“ konfrontiert: Auf spielerische Art sollen die Kinder die dialogisch-ethische Konzeption des Philosophen Emmanuel Lévinas kennenlernen. Lévinas hatte uns auf die tiefe Bedeutung der AntlitzSprache aufmerksam gemacht, als er die „Offenbarung“ des Gesichts eines anderen Menschen beschrieb. Die Kinder werden gemeinsam mit mir darüber nachdenken, wie wir mit dem Gesicht der Anderen umgehen - mit dem Gesichtsausdruck, mit den Emotionen, positiven wie negativen, die das Gesicht zeigt, mit den Augen der Anderen und mit dem Augenkontakt. Kurz wird auch die Sprache ein Thema sein: Wodurch wird unsere Rede zu einem Gespräch? Wodurch ein Gespräch zum Dialog? Was bedeutet der Zusammenhang „WortAntwort"? Unter welchen Voraussetzungen wird das „Andere“ zum „Fremden“ und das „Fremde“ zum „Feindlichen“? Was sagt die Philosophie über unsere alltägliche Sprache? Wie kann diese dialogisch sein? Als letztes Beispiel des Dialogverstehens wird ein Gedanke des Philosophen Józef Tischner bearbeitet, nämlich dessen Solidaritätsbegriff. Die Kinder werden darüber nachdenken, was es heißt, solidarisch zu sein, im Gefüge des „Wir“ zu leben. 


\section{Gliederung des Workshops}

\subsection{Integrationsspiele - Teil I}

Zur Begrüßung biete ich den Kindern ein Spiel an, bei dem sich die Kinder

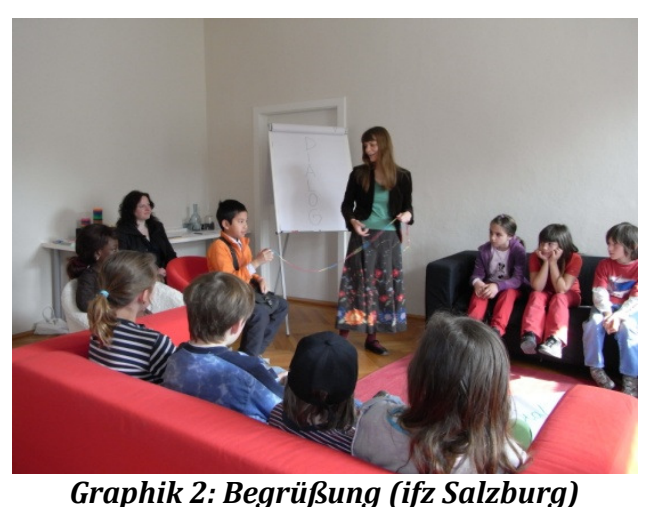

Graphik 2: Begrüßung (ifz Salzburg) untereinander kennenlernen können. Thematisch gesehen stellen solche Spiele eine Einleitung in die dialogische Problematik dar.

\subsection{1 "Wollknäuel"}

Die Kinder sitzen im Kreis, die Referentin hat einen Wollknäuel, den sie einem Kind zuwirft; gleichzeitig hält sie das Fadenende fest. Das Kind, das von der Referentin den Wollknäuel bekommen hat, muss jetzt den Namen der Referentin wiederholen und seinen eigenen laut sagen. Die Kinder werfen sich den Wollknäuel zu, halten aber immer den Wollfaden in der Hand, sodass inmitten des Kreises eine Art Spinnengewebe - eine Vernetzung - entsteht; jedes Kind also muss den Namen der Person sagen, von der es den Wollknäuel bekommen hat, und dann sich selbst vorstellen, bevor es den Wollknäuel weiterwirft. Zum Schluss kommt der Wollknäuel zurück zur Referentin. Diese schneidet die Wolle zwischen den Kindern so ab, dass jedem Kind ein Stück Wolle

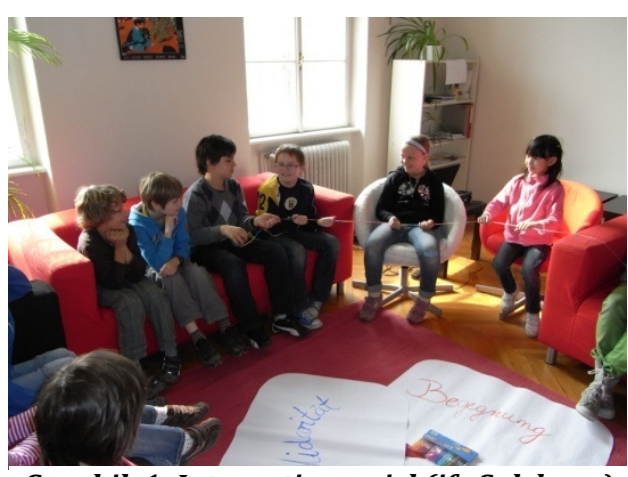

Graphik 1: Integrationsspiel (ifz Salzburg) bleibt. Jedes Kind kann jetzt sein Stück behalten und soll nicht vergessen, von wem es die Wolle bekommen und wem sie sie weitergegeben hat.

In einem kurzem Gespräch kommt eine Zusammenfassung dieses Spiels: Wir haben verschiedene Namen, die können sich wiederholen, die können aus verschiedenen Sprachen stammen, die können auch etwas bedeuten etc. Die Namen sind das, was uns identifiziert, und zugleich das, was uns unterscheidet. Was identifiziert uns, wodurch unterscheiden wir uns noch?

Dauer: bis 8 Min.

\subsection{2 "Augenkontakt"}

Die Kinder arbeiten in Paaren. Sie sitzen sich gegenüber und haben die Aufgabe, sich ohne Augenkontakt kurz umzuschauen. Danach sollen sich die 


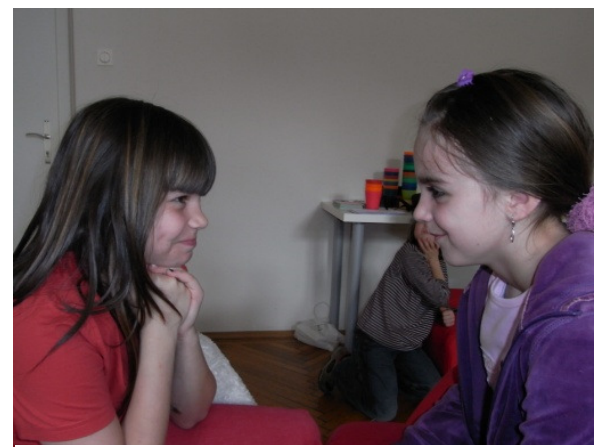

Graphik 3: „Augenkontakt“ (ifz Salzburg)

Kinder gegenseitig genau betrachten und zum Ende einen Augenblick lang in intensivem Augenkontakt verweilen.

Zum Schluss kommt ein kurzes Gespräch über die Gefühle und Emotionen, die wir dabei gespürt haben: Was sehen wir am Anderen? Wie kann man eine andere Person ignorieren? Haben wir uns geschämt? Wie fühlt man sich, wenn man von jemandem ignoriert wird? Welche Spannungen entstehen? Folgende Begriffe werden dabei geklärt: ignorieren, distanzieren, jemanden ausschließen, jemanden wahrnehmen, tolerieren, akzeptieren.

Dauer: bis 5 Min.

\subsection{Thematische Übungen - Teil I}

Einstieg in die Dialogphilosophie.

\subsection{1 "Gesicht"}

Diese Übung ist eine Weiterführung der Idee aus dem Spiel „Augenkontakt“. Ich lege 4-5 großformatige Porträtfotos auf den Boden. Es sind Fotos von Menschen aus verschiedenen Ländern und verschiedenen Alters. Ich zeige ein Foto von Emmanuel Lévinas und erzähle kurz von diesem französischen Philosophen: In seinen Büchern und in seinen Vorträgen hat er gesagt, dass das menschliche Gesicht „zu uns spricht“. Was konnte er damit meinen? Was sagen uns die Gesichter, die wir auf den Fotos sehen?

Dauer: ca. 8 Min.

\subsection{2 "Sprache eines Gesichts"}

Die Kinder arbeiten in Kleingruppen. Jede Gruppe bekommt ein großes Blatt Papier und bunte Stifte. Die Kinder sitzen in jeder Gruppe in einem kleinen Kreis, jedes Kind zeichnet ein Element des Gesichts und gibt das Papier weiter. So wandert das Blatt etwa 2-3 Runden im Kreis, bis ein Porträt fertig ist. Dann präsentieren die Kinder ihre Zeichnungen vor den anderen Gruppen. Die jeweiligen Gruppenmitglieder sagen, was die gezeichneten Gesichter sprechen. Was wollten sie zeichnen? Was ist entstanden und was sehen sie auf den anderen Porträts? Zum Schluss fragt die Referentin die Kinder, ob sie in diesem Gesicht, das sie gezeichnet haben, etwas sehen, was auch ihre eigenen 
Gesichter sagen könnten oder was sie manchmal bei anderen Menschen wahrnehmen.

Dauer: ca. 10 Min.

\subsection{3 "Dialog aufbauen"}

Die Referentin beginnt mit einer Frage: „Was bedeutet 'Dialog', wer kennt dieses Wort?" Auf einem Plakat werden die Beiträge der Kinder notiert. Die Referentin schreibt das Wort „Dialog“ vertikal an die Tafel und fragt die Kinder, ob sie wissen wollen, welche freien Gedanken sie selbst zu diesem Wort hat. Ein Beispiel, das an der Tafel entstehen kann:

$$
\begin{aligned}
& \text { Dominik } \\
& \text { mIteinander } \\
& \text { lAut? } \\
& \text { GeduLd } \\
& \text { TelefOn } \\
& \text { Gespräch }
\end{aligned}
$$

Dazu erkläre ich: „Dominik ist mein Freund, wir reden gern miteinander. Kann ein Dialog laut sein? Manchmal brauche ich viel Geduld, dass ich im Dialog bleibe oder dass ich nicht laut werde. Dialog ist ein Gespräch. Wie und wo kann man gut miteinander sprechen? Im Kreis, zu Hause, am Spielplatz, am Telefon... Wie noch, wo

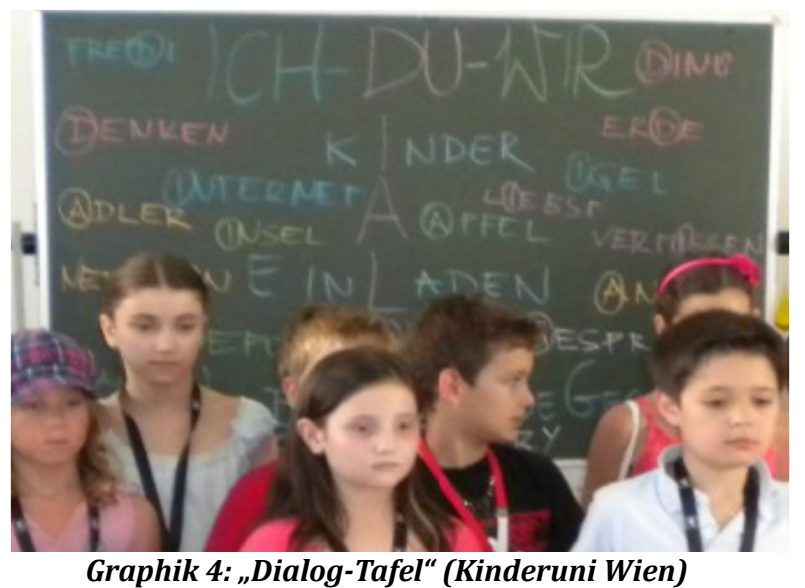
noch?" Dann werden die Beispiele an der Tafel gelöscht und die Kinder aufgefordert, nach anderen Assoziationen zu suchen.

Dauer: ca. 10 Min.
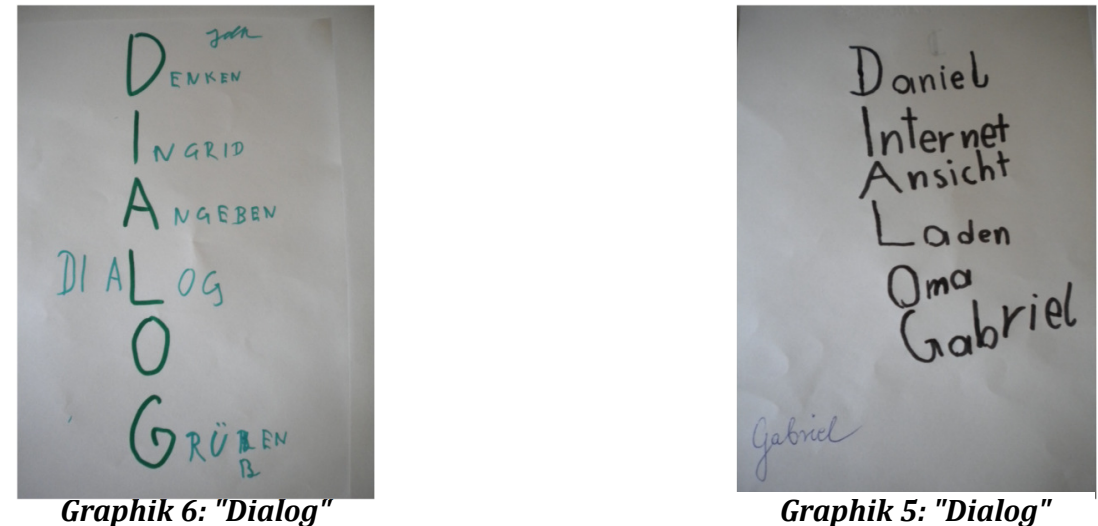


\subsection{Vortrag (PPP) und Diskussion}

Ich werde nun den Kindern erzählen, dass die Philosophie, die an den Schulen oder an den Universitäten unterrichtet wird, sich mit vielen verschiedenen Themen beschäftigt: Wer ist ein Philosoph? Jemand, der über die Welt, die Natur, über die Menschen nachdenkt. Unser Thema ist heute die Philosophie des Dialogs. Diese philosophische Richtung beschäftigt sich mit den menschlichen Relationen, Beziehungen, Begegnungen, deswegen heißt sie dialogische Philosophie oder Begegnungsphilosophie.

\subsubsection{PPP: „Ich-Du-Wir"}

Graphiken 7-24 (Seiten in PPP: 1-18): „Ich-Du-Wir“
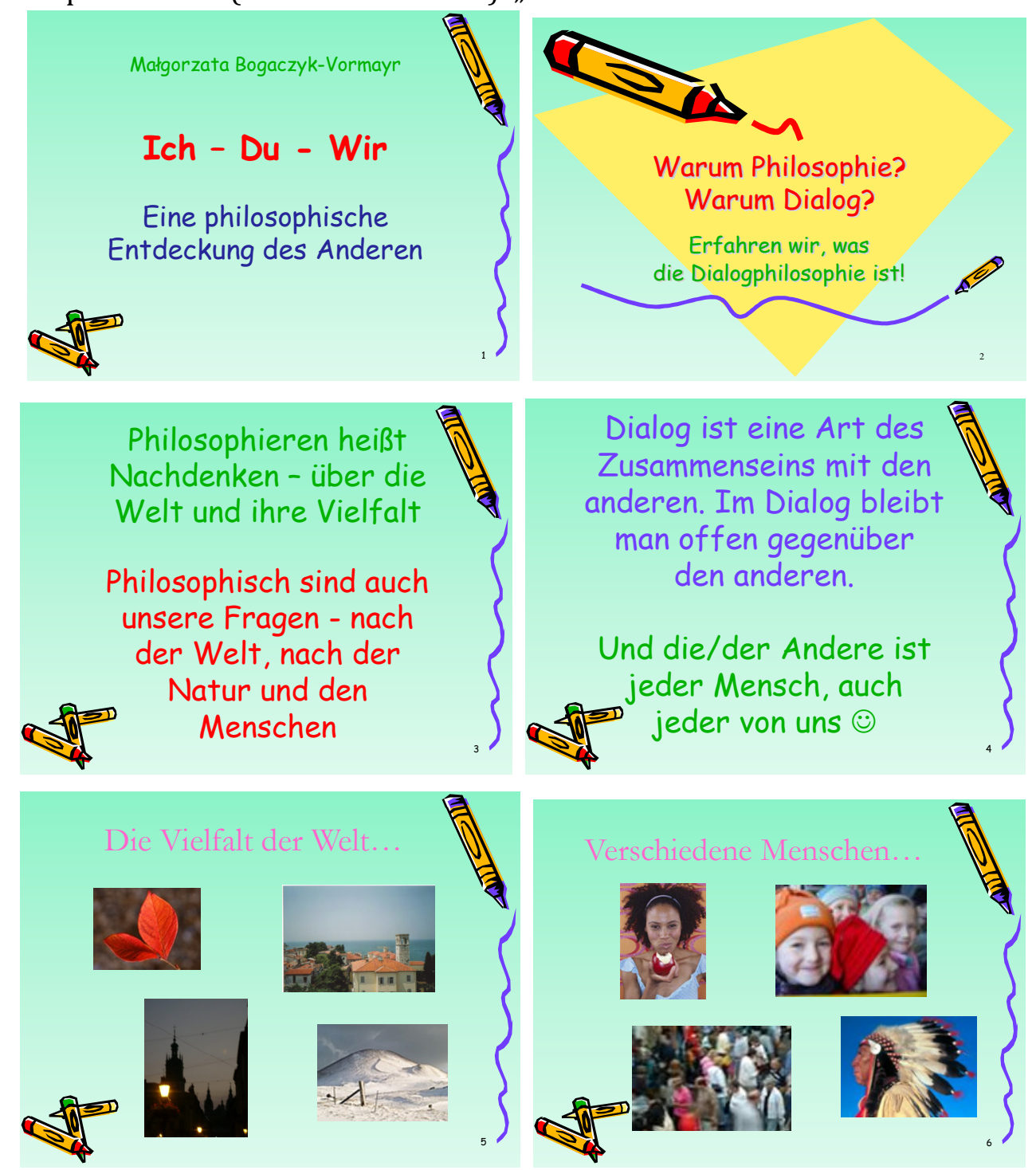


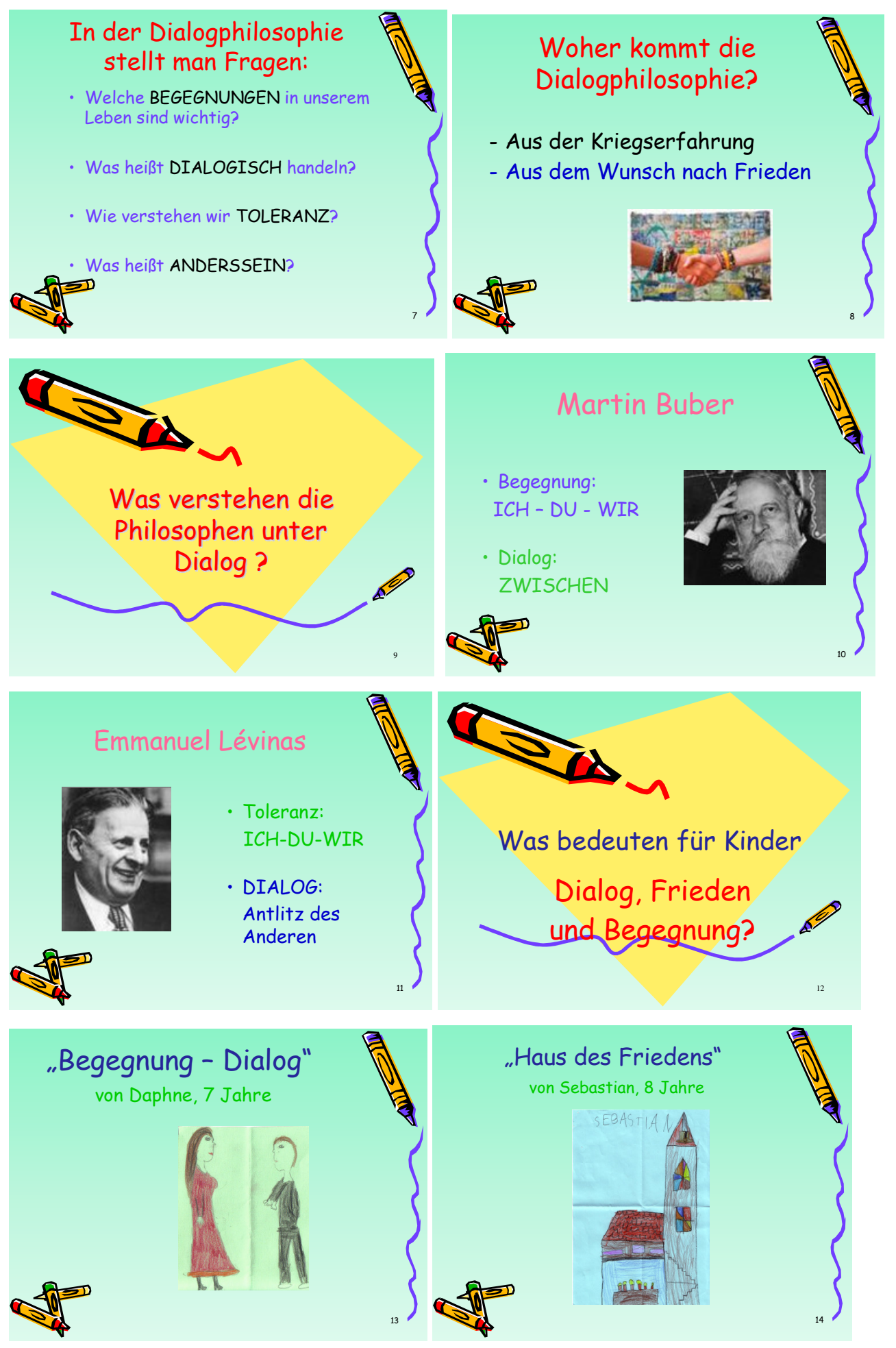




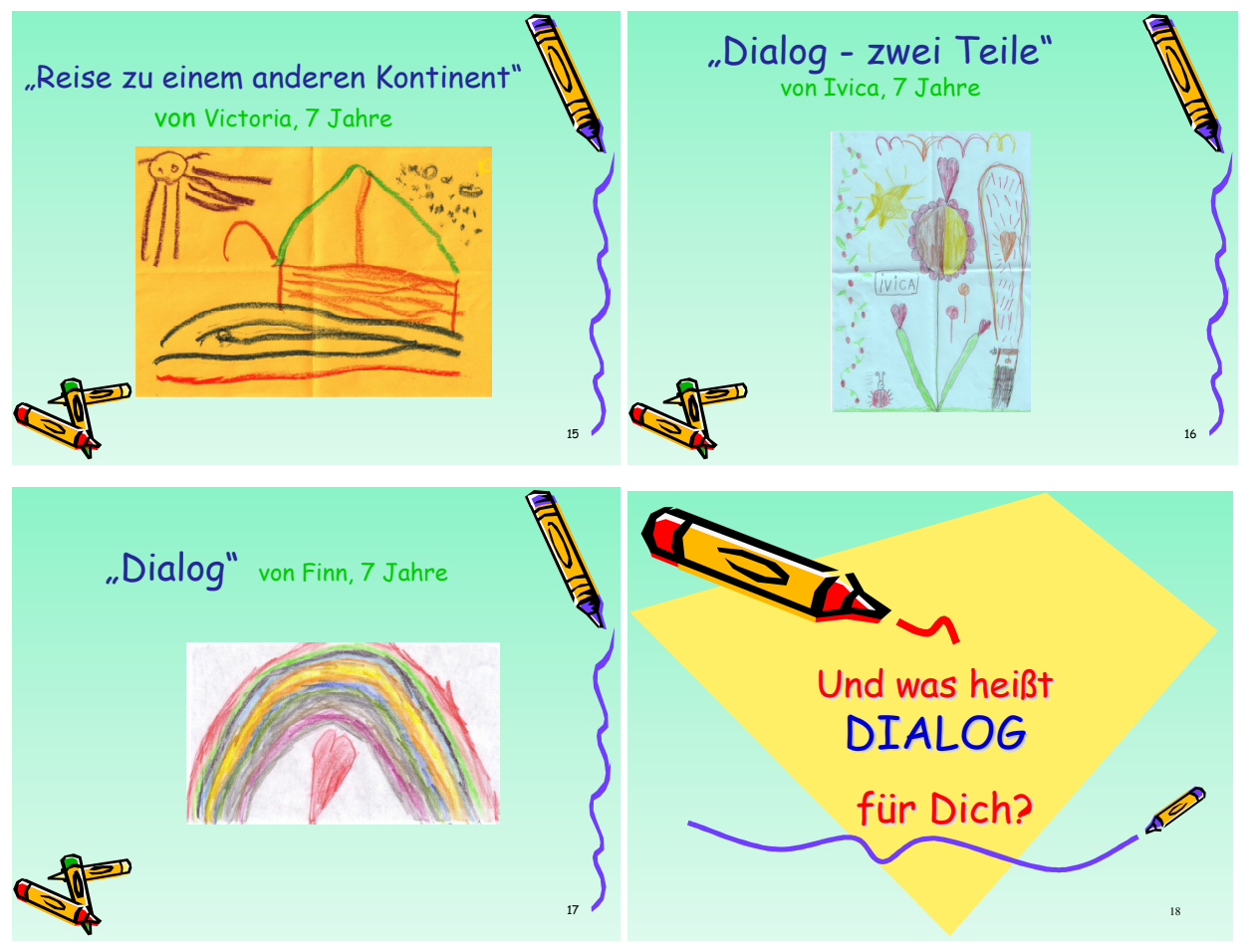

Dauer: ca. 15 Min.

\subsubsection{Diskussion:}

Nach der Präsentation hänge ich noch drei Plakate an die Wand - auf jedem steht ein Zitat, illustriert mit dem Foto und dem Namen jenes Philosophen, von dem diese Worte stammen: 1) „Wer ,Du' spricht, hat kein Etwas, hat nichts. Aber er steht in der Beziehung. Alles wirkliche Leben ist Begegnung." (Martin Buber); 2) „Einen Dialog zu beginnen heißt Geben und Bekommen. Weil wir in einem Dialog voneinander lernen.“ (Emmanuel Lévinas); 3) „Es gibt keine Solidarität ohne Dialog. Es gibt kein ,Wir' ohne Dialog. Und 'Wir' sind wir nicht nur für 'Uns', sondern auch für die anderen." (Józef Tischner).

Dazu kommen Erläuterungen, Fragen und ein Gespräch darüber: Wir leben mit materiellen Dingen, inmitten der Natur und unter Menschen. „Viel auf der Welt können wir besitzen, z.B. die Dinge“, sagte der Philosoph Martin Buber, „aber nicht die Menschen.“ Zwischen Menschen entstehen verschiedene Beziehungen und Relationen. Welche kennen wir? In der Klasse, mit einer Freundin, zu Hause, mit dem Opa, mit der Schwester... Das nennt der Philosoph Martin Buber jemanden als ein ,Du' kennen lernen, jemanden nicht als Ding, sondern als Person zu behandeln. Das bedeutet auch, jemandem nahe zu kommen - und das ist es, was unser Leben schön macht. Was machen wir bei einer Begegnung? Miteinander reden, spielen, lernen - was mögen die Kinder? Bekommen wir beim Spielen, im Gespräch etwas von den anderen? Was geben wir? Was bedeutet es, dass wir nicht nur "für uns" sind? Und wann können wir einen Dialog beginnen? Ist das in einem Streit möglich? Ist ein solches Gespräch, in dem jede und jeder die eigenen Gedanken immer 


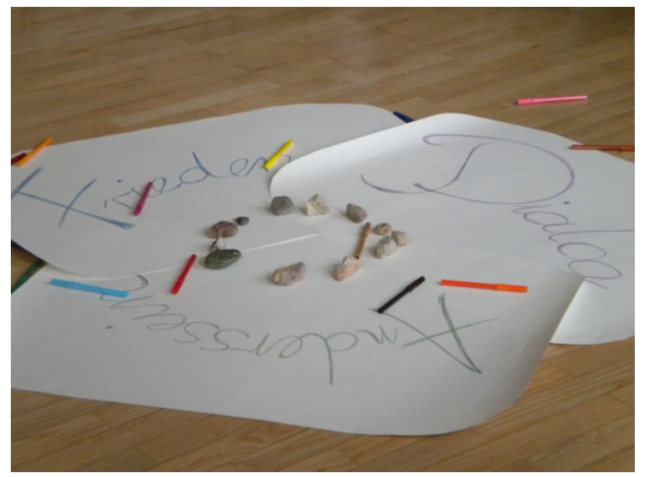

Graphik 25: „Philosophische Begriffe 1“(Kinderuni Salzburg)

sagen darf und alle sich gegenseitig immer zuhören, überhaupt möglich und richtig?

Während der Diskussion schreibe ich auf ein Arbeitsblatt alle Begriffe, die ich verwende, und wiederhole diese immer wieder: „Dialog“, „Anderssein“, „Solidarität“... Daneben notiere ich die Gedanken, die von den Kindern kommen, wie z. B. „Gespräch“, „reden“, „sich treffen“, „zusammen sein“.

Dauer: ca. 15 Min.

\subsection{Thematische Übungen - Teil II}

\subsection{1 "Begriffe - begreifen"}

Jedes Kind bekommt zwei kleine Zettel. Die Kinder sollen ihre eigenen Assoziationen oder Bemerkungen zu den Begriffen, welche auf den Arbeitsblättern zu lesen sind - z.B. „Dialog“, „Solidarität", „Frieden“ -, etwas aufschreiben bzw. zeichnen. Dann zeigt jedes Kind seine Arbeit allen anderen und entscheidet selbst (oder wenn es will, mit Hilfe der anderen Kinder), wohin genau seine Aufzeichnungen gehören.

Dauer: bis 10 Min.

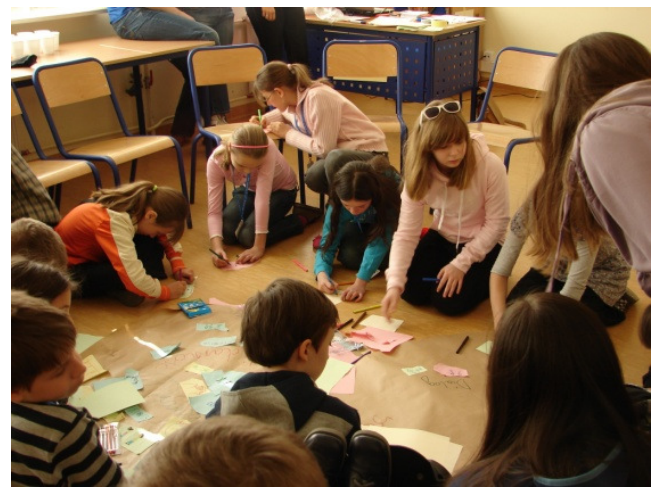

Graphik 26: „Philosophische Begriffe 2“ (Kinderuni Warszawa)

\subsection{1 "Kreuzworträtsel in Bewegung"}

Die Kinder arbeiten in Kleingruppen. Sie bekommen ein Kreuzworträtsel mit 6-8 Fragen (z.B.: „Jeder ist...", „Wie sagt man in einer philosophischen Sprache, wenn man jemanden zu einem Dialog trifft?"). Die Kinder können diese Fragen selbst beantworten oder ein im Raum befindliches Kuvert mit der jeweils richtigen Antwort auffinden (z.B.: „Jeder ist ANDERS“, „BEGEGNEN“). Wichtige Spielelemente neben der gemeinsamen Arbeit sind hier also das Arbeitstempo und die Bewegung.

Dauer: bis 5 Min. 


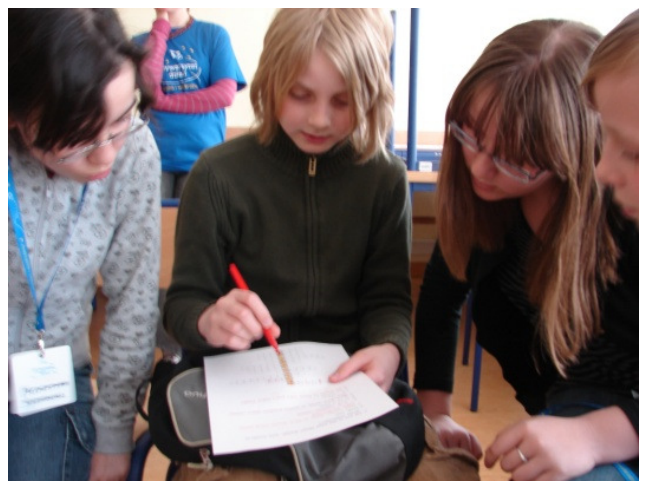

Graphik 27: Kreuzworträtsel in Bewegung“ (Kinderuni Warszawa)

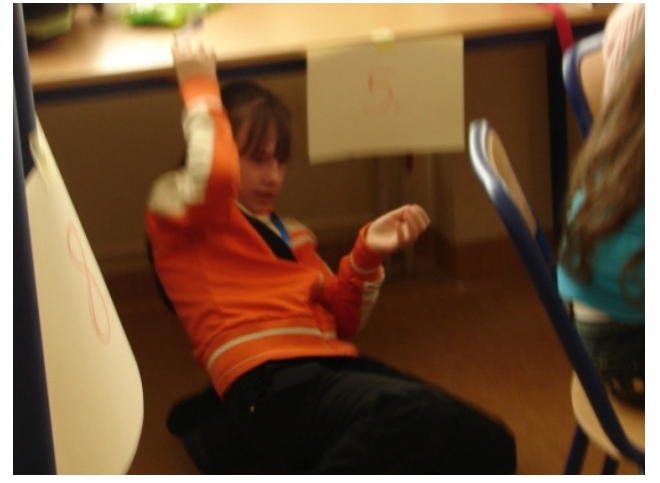

Graphik 28: Kreuzworträtsel in Bewegung“ (Kinderuni Warszawa)

\subsection{2 "Unterschiede"}

Die Kinder sitzen im Kreis. Jedes Kind sagt etwas über sich selbst und über eine Person, die neben ihm sitzt. Die Aufgabe besteht darin, über die Unterschiede zwischen zwei nebeneinander sitzenden Menschen auf eine positive bzw. neutrale Art zu reden, z.B.: „Anna geht in die zweite Klasse, anders als ich." Wenn ein Kind will, kann es gern neben den bestehenden Unterschieden auch eine Ähnlichkeit betonen. Bei der Erklärung der Regeln ist es wichtig, die Kinder anzuregen, dass sie (ev. unter Mithilfe der Referentin) möglichst ausführliche Sätze bilden, z.B.: „Tom hat braune Haare, ich habe blonde und kürzere Haare. Ich trage einen Pullover und Tom ein T-Shirt", „Marias beste Freundin heißt Eva, meine beste Freundin ist Kathrin, aber wir spielen manchmal zu viert."

Ich bringe sodann eine kurze Zusammenfassung: Wenn wir jemanden mögen, sprechen wir oft davon, was uns gemeinsam ist oder worin wir uns ähnlich sind. Wenn wir von Unterschieden sprechen, beurteilen wir oft, was besser ist, was uns besser gefällt. Heute lernen wir, dass es etwas Positives ist, wenn man anders ist: anders spricht, anders denkt, andere Träume und Meinungen hat. Die Dialogphilosophen glauben, dass das Andere, das Unterscheidende vor allem eine schöne Bedeutung hat, es ist ein großer und positiver Wert des Menschen.

Dauer: bis 10 Min.

\subsection{Abschlussspiele}

\subsection{1 "Ein geheimes gutes Wort"}

Dieses Spiel ist eine Wiederholung der Übung „Dialog aufbauen“. Jedes Kind bekommt ein A4-Blatt, auf welchem sein Name (Anna, Christoph, Tobias etc.) vertikal geschrieben wurde. Die Kinder tragen die Zettel auf dem Rücken. Jede_r darf einem anderen Kind ein gutes Wort auf den Rücken schreiben ein Wort, das auf eine gute Art diese Person beschreibt (ihre Hobbys, Fähigkeiten, Talente, Eigenschaften). Ein Beispiel dafür: 


\section{Lustig \\ zeIchnen \\ Nett \\ Dinosaurier \\ tAnzen}

Dauer: ca. 6 Min.

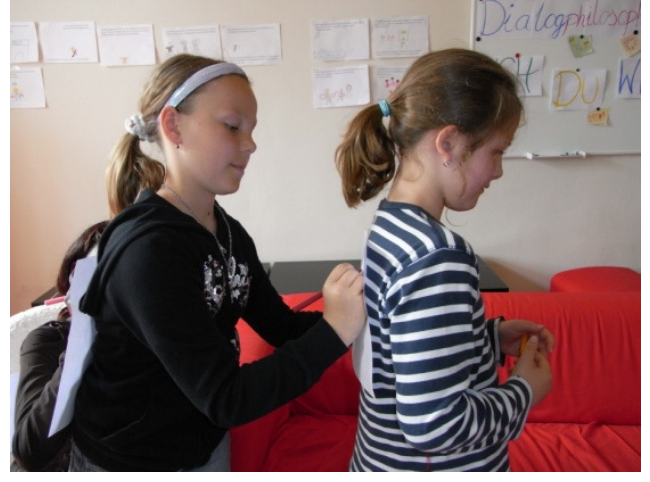

Graphik 29: „Ein gutes Wort“ (ifz Salzburg)

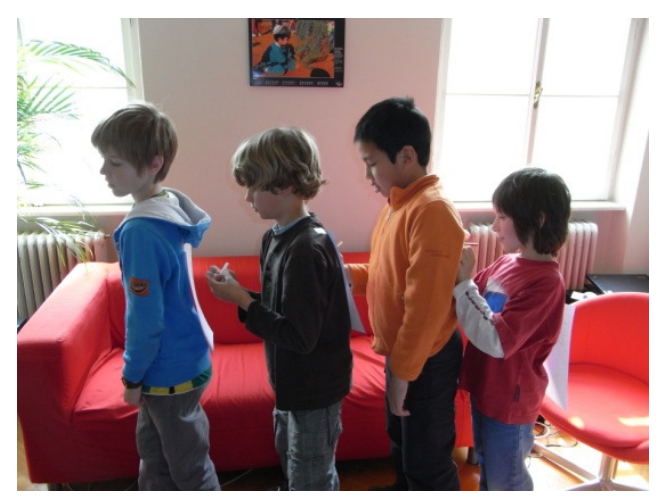

Graphik 30: „Ein gutes Wort“

\subsection{2 "Wasser schenken, Gutes sagen"}

Die Kinder bekommen halbvolle Plastikbecher. Ihre Aufgabe ist es, herumzugehen und bei jedem anderen Teilnehmer kurz stehenzubleiben. Die Kinder sagen sich gegenseitig etwas Nettes - sie drücken ihre Sympathie aus, ihre Akzeptanz, sagen ein Kompliment, und als Symbol der Sympathie schenken sie dem Anderen etwas Wasser aus dem eigenen Becher. Wichtig ist, dass jedes Kind möglichst allen anderen Kindern auf diese Art BEGEGNET, dass die Kinder auch gern das Wasser „weiterschenken“.

Dauer: bis 8 Min.

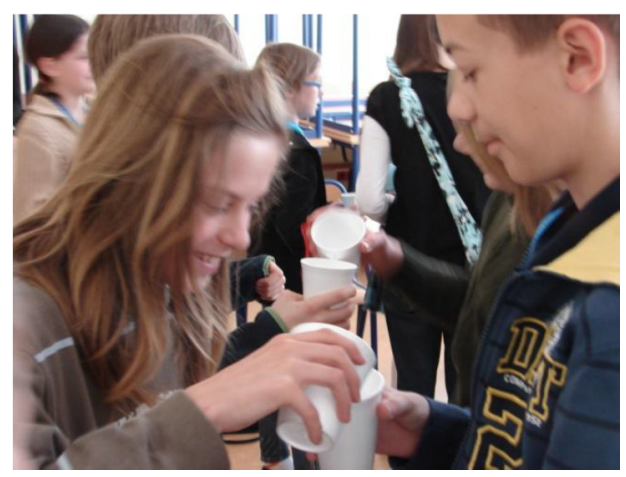

Graphik 31: „Wasser schenken..." (Kinderuni Warszawa)

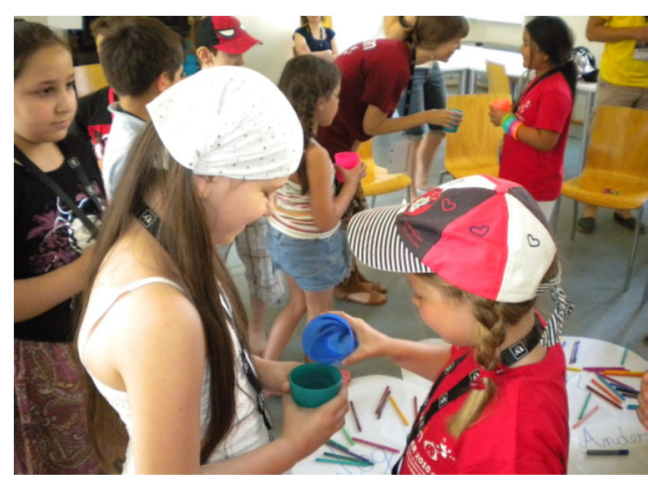

Graphik 32: „Wasser schenken..." (Kinderuni Wien)

Vorgeschlagene Gesamtzeit: 100 bis 120 Min. 


\section{Literature}

Buber, M. 1979, "Ich und Du". In: Ders., Das dialogische Prinzip, Heidelberg: Verlag Lambert Schneider.

Belke, F. 1963, "Dialogischer und pädagogischer Bezug in Martin Bubers Konzeption des Relationalen". In: Vierteljahresschrift für wissenschaftliche Pädagogik, Jg. 39, H. 4, 265-299.

Bollnow, O.F. 1982, "Das Problem der Begegnung”. In: Alma Mater Aquensis , Jg. 19 (1981/1982, 75-84.

Gerner, B. (Hg.) 1974, Martin Buber - pädagogische Interpretation zu seinem Werk. München: Ehrenwirth.

Göbling, H.J. 1995, "Die Fremdheit des Anderen - Grund oder Abgrund pädagogischer Verantwortung?". In: Pädagogische Rundschau, Jg. 49, 631-643.

Lévinas, E. 1996, Ethik und Unendliches: Gespräche mit Philippe Nemo, übers. von D. Schmidt. Wien: Passagen-Verlag.

Tischner, J. 1982, Ethik der Solidarität: Prinzipien einer neuen Hoffnung. Graz/Wien: Verlag Styria.

Waldenfels, B. 1997, Topographie des Fremden, Studien zur Phänomenologie des Fremden. Frankfurt am Main: Suhrkamp. 
Małgorzata Bogaczyk-Vormayr (Poznań)

\title{
Workshop in Kinderphilosophie: Ich-Du-Wir. Eine philosophische Entdeckung des Anderen
}

\begin{abstract}
Die Autorin präsentiert ihr Konzept für einen Workshop in Kinderphilosophie, in welchem sie eine theoretische Darstellung der Problematik der Begegnungsphilosophie - in einem pädagogischen Kontext sowie eine detaillierte Gliederung des Workshops darstellt. Die Teilnehmerinnen und Teilnehmer dieses Workshops sollen die philosophischethische Problematik des Anderen kennenlernen, sie werden dazu befähigt, die folgenden dialogphilosophischen Begriffe zu unterscheiden und inhaltlich abzugrenzen: das Andere, die/der Andere, Dialog, Begegnung, Relation, Solidarität. Die Autorin präsentiert hier, wie die Denkweisen einiger Philosophen auf spielerische Art den Kindern nähergebracht werden (Martin Buber, Emmanuel Lévinas, Józef Tischner). Die Kinder sollen somit erfahren, was die Begegnungsphilosophie beinhaltet, aber vor allem werden die mit den Phänomenen der Alterität konfrontiert. Das Ziel ist es, die soziale und kulturelle Akzeptanz der Kinder für den Anderen zu vertiefen.
\end{abstract}

Keywords: Kinderphilosophie, Dialog, Begegnung, Akzeptanz, Solidarität, Friedenserziehung, soziales Lernen, Interkulturalität

Ethics in Progress (ISSN 2084-9257). Vol. 4 (2013). No. 1. pp. 106-118. 